\title{
A distributed decision method for missiles autonomous formation based on potential game
}

\author{
JIA Xiang ${ }^{1, *}$, WU Sentang ${ }^{2}$, WEN Yongming ${ }^{2}$, and YAO Zheng ${ }^{2}$ \\ 1. Beijing Institute of Electronic Engineering, Beijing 100854, China; \\ 2. School of Automation Science and Electrical Engineering, Beihang University, Beijing 100191, China
}

\begin{abstract}
The distributed cooperative decision problems of missiles autonomous formation with network packet loss are investigated by using the potential game based on formation principles. In particular, a dynamic target allocation method for missiles formation is provided based on the potential game and formation principles, after the introduction of cooperative guidance and control system of the missiles formation. Then we seek the optimization of a global utility function through autonomous missiles that are capable of making individually rational decisions to optimize their own utility functions. The first important aspect of the problem is to design an individual utility function considering the characteristics of the missiles formation, with which the objective of the missiles are localized to each missile yet aligned with the global utility function. The second is to equip the missiles with an appropriate coordination mechanism with each missile pursuing the optimization of its own utility function. We present the design procedure for the utility, and present a coordination mechanism based on spatial adaptive play and then introduce the idea of "cyclical selected spatial adaptive play" and "negotiation based on time division multiple address (TDMA) protocol formation support network". Finally, we present simulations for the distributed dynamic target allocation on the comprehensive digital simulation system, and the results illustrate the effectiveness and engineering applicability of the method.
\end{abstract}

Keywords: distributed decision, dynamic target allocation, missiles autonomous formation, potential game.

DOI: $10.21629 / J S E E .2019 .04 .11$

\section{Introduction}

The method of cooperative decision is generally divided into centralized decision, centralized-distributed decision and distributed decision according to the decision mechanism [1]. Centralized decision can ensure consistency and optimality of the results, but it has the following disadvantages when the formation scale is large: the amount of computation and communication is large, it cannot guaran-

\footnotetext{
Manuscript received November 27, 2017.

*Corresponding author.

This work was supported by the Industrial Technology Development Program (B1120131046).
}

tee the robustness and stability of the system in the condition of uncertainty information or serious packet loss, etc. The centralized-distributed decision is between the centralized decision and the distributed decision, such as hierarchical decision, so this kind of methods also has the same problems as centralized decision in a certain degree. Distributed decision can not only ensure that each formation member obtains a viable solution which guarantees the local optimization in the condition of uncertainty environment and limited amount of computation and communication, but also take the global optimization into consideration to a certain extent. However, the proof of the consistency and optimization for the selection results of distributed decision are difficult.

These years, a lot of researches [2-8] on the distributed decision and control problem have been carried out. For example, Olfati-Saber [2] focused on "kinetic" coordination and strategic coordination, Ahuja et al. [3] concentrated on heuristic methods to quickly obtain near optimal assignment in relatively large instances.

The distributed decision based on potential game [410] is a decision model that takes into account the selfishness of individual utilities and the integrity of group utility which could solve the problem of incomplete information of the distributed system, stimulate the autonomy of the individual in the uncertain environment, and ultimately make the whole to be optimal gradually through the advantages of individual choice.

This method has been adopted in several researches. Marden et al. [8] used the potential function optimized by coordination decisions of local objective functions to capture the global objective of multi-agent systems. Arslan et al. [10] used a potential game to coordinate the global objective function and the local objective functions of rational agents. Li [11] developed a game theoretic formulation for multiple unmanned aerial vehicle (UAV) cooperative search and surveillance. Marden [12], Johari et al. [13], Young et al. [14] and Li et al. [15] respectively introduced 
several methods into the potential game to ensure the efficiency of the resulting equilibrium.

Compared with the previous research, this paper is characterized by the combination of the potential game and missile formation principles, with a consideration of communication networking protocol and network delay. In this way the algorithm is closer to practical application and more suitable for missiles autonomous formation (MAF).

\section{Background}

The distributed cooperative decision method discussed in this paper is the key to study the decision and management system of MAF. The MAF decision and management system is an important part of MAF cooperative guidance and control technology. In this paper, we take the MAF [1620] for example to introduce the proposed method.

MAF cooperative guidance and control technology refers to the problem of autonomous formation to perform the task during the flight from the launch of the missiles to the terminal guidance stage, including the midcourse and the handover guidance. Its architecture mainly includes five parts [16-18] (see Fig. 1):

(i) Information acquisition system (IAS);

(ii) Decision and management system (DMS);

(iii) Flight control system of MAF (FCSM);

(iv) Member flight control system (MFCS);

(v) Support networks system (SNS).

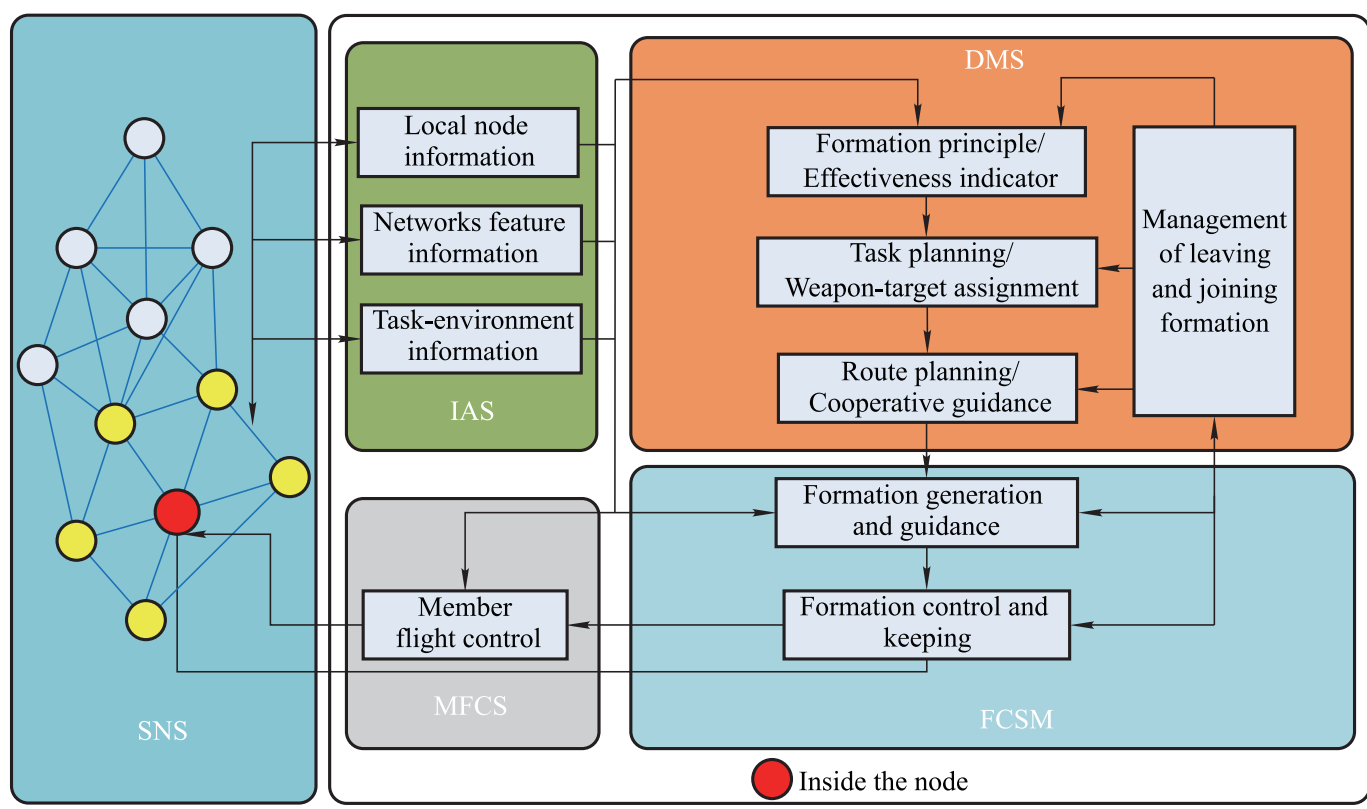

Fig. 1 Architecture of the MAF cooperative guidance and control system

\section{Potential game}

These years the potential game was used in the sensor networks [21-25] and multi-agent [26-29], while use of the potential game for distributed decision is focused in this paper, and the modeling mechanism is as follows.

Assume that there are $n_{v}$ unmanned platforms to allocate $n_{t}$ targets. Each target and the unmanned platform have their own characteristics. $\nu_{1}, \ldots, \nu_{n_{v}}$ are $n_{v}$ unmanned platforms, while $T_{0}, T_{1}, \ldots, T_{n_{t}}$ denote $n_{t}$ targets, where $T_{0}$ represents a virtual target: "empty target". The set of targets that can be assigned to the unmanned platform $\nu_{i}$ is denoted as $\boldsymbol{A}_{i} \subset \boldsymbol{T}$. Thus, the target assigned to the unmanned platform $\nu_{i}$ at a given time can be denoted as $a_{i} \in \boldsymbol{A}_{i}$, and then the set $\boldsymbol{a}=$ $\left(a_{1}, \ldots, a_{i}, \ldots, a_{n_{v}}\right)$ is a task target allocation plan for a whole group at the given time.

The global utility function is denoted by $U_{g}(\boldsymbol{a})$, and the individual utility function of the unmanned platform $\nu_{i}$ is denoted by $U_{v_{i}}(\boldsymbol{a})$. Each member of the formation chooses its own target according to the basic rules of the group, in their own optional set of targets, to maximize its own utility. However, the setting of the individual utility function is usually corrected following the optimization of the global utility. In the process of distributed decision, each individual will select the target under the pre-set coordination mechanism. Only when the selection of individual and global utility functions satisfies the specific requirements, the optimization and coordination process shows as a convergence process of the potential game or the generalized potential game. In this way, the process will eventually reach the Nash balance. 
A generalized potential game forms when the referred individual utility functions and the global utility function can satisfy the following formula [7-9]:

$$
\begin{gathered}
U_{v_{i}}\left(a_{i}^{\prime}, a_{-i}\right)-U_{v_{i}}\left(a_{i}^{\prime \prime}, a_{-i}\right)>0 \Leftrightarrow \\
U_{g}\left(a_{i}^{\prime}, a_{-i}\right)-U_{g}\left(a_{i}^{\prime \prime}, a_{-i}\right)>0
\end{gathered}
$$

where $a_{i}^{\prime}, a_{i}^{\prime \prime} \in \boldsymbol{A}_{i}, a_{-i} \in \boldsymbol{A}_{-i}$, and $\boldsymbol{A}_{-i}=\boldsymbol{A}_{1} \times \boldsymbol{A}_{2} \times \cdots$ $\times \boldsymbol{A}_{i-1} \times \boldsymbol{A}_{i+1} \times \cdots \times \boldsymbol{A}_{n_{v}}$.

The selection of the utility function of the potential game and the setting of the coordination mechanism will directly affect the convergence rate and the quality of the balance point. The proof of the convergence and the quality of the balance point is usually the difficult part for the game theory applied in engineering.

\section{Distributed decision problem description of MAF}

The distributed cooperative decision of the MAF usually comes with the following problems: high dynamic and uncertainty, huge communication costs, local and global information structure difference, decentralization of control and implementation. In addition, cooperative distributed decision has optimal or better results only in moderately complex operating scenarios, so sometimes the optimal solution cannot be obtained or the feasibility of the solution cannot be guaranteed, especially the information obtained by the communication or computing with long delay, strong noise and the high bit error rate.

It will result in waste of resources or even lead the system into the local optimal errors when thoughtlessly adopting the distributed decision-making or blindly using a certain individual advantage function or coordination method for different environments and situations. Therefore, for different mission environments and battlefield situations, the distributed decision strategy of the MAF needs to be adjusted. For example, the analytical solution of the optimal problem is necessary for a small scale formation. However, in the case of high environmental complexity, random and mixed strategies can be used to suppress the diffusion of error information and enhance the robustness of the system. The adjustment of the decision strategy will also directly affect the complexity of the calculation, the decision instantaneity, the degree of optimization, etc. In this way, the principle of autonomous formation will be an important basis for selecting decision methods and guiding the decision process.

The formation principle can be summarized as three major aspects: the principle of necessity for group cooperation, the principle of maximum comprehensive benefit and the principle of returning patronage to every member of the group [16]. (i) The principle of necessity for group cooperation

Multi-missiles will constitute a cooperative formation only if they can obtain significantly improved comprehensive combat effectiveness as a formation rather than as an individual missile or multi-missiles without cooperation when carrying out a certain mission. The principle indicates the necessity to make the multi-missiles work as cooperative formation while carrying out a mission.

(ii) The principle of maximum comprehensive benefit

The maximum comprehensive benefit for a formation embodies the optimal balance between the individual benefit and the combat effectiveness of the formation, and also between the cost and the effectiveness. In other words, the goal of the cooperative formation is to maximize the comprehensive combat-effectiveness.

(iii) The principle of returning patronage to every member of the group

It is needed to fully take into account the different performances and benefit of missile individuals, when a missiles formation carries out a mission in a cooperative manner, and all missile members should be taken into consideration during the process of information acquisition and sharing, decision making and concerted action. Thus it is a basic requirement of cooperative formation to be inclusive for all members of the integrity formation.

The decision of the MAF needs to ensure the instantaneity and reliability, but a completely individual spontaneous coordination process will greatly affect the speed of optimization. Thus the combination of the principle of autonomous formation and the potential game can make full use of the individual's intelligence, and adjust the decisions from the macro based on the task environment, and finally make the formation achieve the Nash balance. And then the potential game can be constructed based on formation principles with the following characteristics.

(i) Necessity for group cooperation

The choice of coordination mechanism and individual utility function needs to be based on the principle of necessity for group cooperation, the combat mission of the MAF and the natural environment (such as group instantaneous scale, current communication quality, coordinated guidance and control capability).

(ii) Maximum comprehensive benefit

The global utility function, individual utility function and coordination mechanism need to meet the combat mission according to the principle of maximum comprehensive benefits.

(iii) Returning patronage to every member of the group

The assignment without the integrity and the patronage will be punished according to the principle of returning pa- 
tronage to every member of the formation. At the same time, the information entropy is added to adjust the randomness of the decision process, which is an important part of the coordination mechanism.

In Section 5 and Section 6, distributed target allocation will be taken as an example of the design of the distributed decision method for MAF based on the potential game and the formation principle.

\section{Individual utility function and global utility function}

Firstly, the global utility function of the potential game is designed, denoted by $U_{g}(\boldsymbol{a})$, according to the three formation principles above. Take the dynamic target allocation as an example, the utility function for the allocation result to the target $T_{j}$ is defined as (2), corresponding to allocation scheme $\boldsymbol{a}=\left(a_{1}, \ldots, a_{i}, \ldots, a_{n_{v}}\right)$.

$$
U_{T_{j}}(\boldsymbol{a})=\max \sum^{m_{T_{j}}} U_{v_{i}-T_{j}}\left(\boldsymbol{v}_{i} \in \nu, T_{j} \in \boldsymbol{T}, a_{i}=T_{j}\right)
$$

where $U_{v_{i}-T_{j}}\left(\boldsymbol{v}_{i} \in \nu, T_{j} \in \boldsymbol{T}, a_{i}=T_{j}\right)$ denotes the dominating value for each missile $v_{i}\left(a_{i}=T_{j}\right)$ to the target $T_{j}$ with the allocation result $\boldsymbol{a}=\left(a_{1}, \ldots, a_{i}, \ldots, a_{n_{v}}\right)$, and $m_{T_{j}}$ denotes the max amount of missiles to destroy the target $T_{j}$. Then the above formula represents the sum of the $m_{T_{j}}$ greatest dominating values of the missiles needed that attack the target $T_{j}$ in the allocation result.

On this basis, the global utility function $U_{g}(\boldsymbol{a})$ is defined as follows:

$$
U_{g}(\boldsymbol{a})=\sum_{T_{j} \in \boldsymbol{T}} U_{T_{j}}(\boldsymbol{a})-\lambda \cdot \sum_{n_{T_{j}}(\boldsymbol{a})=0, T_{j} \in \boldsymbol{T}} \omega_{T_{j}}
$$

where $\lambda \cdot \sum_{n_{T_{j}}=0, T_{j} \in \boldsymbol{T}} \omega_{T_{j}}$ denotes the punishment to the ignoring of the target $T_{j}$ with the threat degree $\omega_{T_{j}}$ in the allocation result, and the variable $\lambda$ is the punishment factor. $n_{T_{j}}(\boldsymbol{a})$ denotes the amount of missiles to attack the target $T_{j}$ in the allocation result.

The choice of individual utility function of potential game generally follows two basic ideas: one is to ensure the relevance of the individual utility function to the global utility function; the other is to ensure the localization of the individual utility function, that is, the use of locally limited information available to build the individual utility function. In addition, it needs to design different individual utility functions for different task environments and natural environments according to the three formation principles. Two kinds of individual utility functions for the dynamic target allocation of the autonomous formation are given, and the task environment and applicable conditions are also given:

(i) Assuming that the formation support network and the information acquisition system can give the complete information, and the communication is perfection, the individual utility function could be

$$
U_{v_{i}}(\boldsymbol{a})=U_{g}(\boldsymbol{a})
$$

At this time, each missile will use the global information obtained to determine the local selection results. However, because of its strong dependence on the network service quality, the amount of calculation and communication will increase sharply when the formation is in a large scale. Therefore, it is only suitable for autonomous formation with good communication status and limited nodes.

(ii) Assuming that the formation support network and the information acquisition system can give information about the neighbor missiles, the individual utility function could be

$$
U_{v_{i}}(\boldsymbol{a})=\sum_{T_{j} \in \boldsymbol{A}_{i}} U_{T_{j}}(\boldsymbol{a})-\lambda \cdot \sum_{n_{T_{j}}(\boldsymbol{a})=0, T_{j} \in \boldsymbol{A}_{i}} \omega_{T_{j}} .
$$

Such an individual utility function requires the locally acquired information that contains all the data needed for calculating the dominating values of all the missiles assigned to each target in the local optional target set $\boldsymbol{A}_{i}$. This individual utility function is designed to reduce the amount of the information and computation, and to meet the problem of distributed decision in uncertain environment. Formula (6) gives the proof that the constructed individual and global utility functions are in line with the general form of the potential game.

$$
\begin{gathered}
U_{g}\left(a_{i}^{\prime}, a_{-i}\right)-U_{g}\left(a_{i}^{\prime \prime}, a_{-i}\right)= \\
\left(\sum_{T_{j} \in \boldsymbol{T}} U_{T_{j}}\left(a_{i}^{\prime}, a_{-i}\right)-\lambda \cdot \sum_{n_{T_{j}}\left(a_{i}^{\prime}, a_{-i}\right)=0, T_{j} \in \boldsymbol{T}} \omega_{T_{j}}\right)- \\
\left(\sum_{T_{j} \in \boldsymbol{T}} U_{T_{j}}\left(a_{i}^{\prime \prime}, a_{-i}\right)-\lambda \cdot \sum_{n_{T_{j}}\left(a_{i}^{\prime \prime}, a_{-i}\right)=0, T_{j} \in \boldsymbol{T}} \omega_{T_{j}}\right)= \\
\left(\sum_{T_{j} \in \boldsymbol{T}, T_{j} \notin \boldsymbol{A}_{i}} U_{T_{j}}\left(a_{i}^{\prime}, a_{-i}\right)-\right. \\
\left.\lambda \cdot \sum_{n_{T_{j}}\left(a_{i}^{\prime}, a_{-i}\right)=0, T_{j} \in \boldsymbol{T}, T_{j} \notin \boldsymbol{A}_{i}} \omega_{T_{j}}\right)- \\
\left(\sum_{T_{j} \in \boldsymbol{T}, T_{j} \notin \boldsymbol{A}_{i}} U_{T_{j}}\left(a_{i}^{\prime \prime}, a_{-i}\right)-\right. \\
\left.\lambda \cdot \sum_{n_{T_{j}}\left(a_{i}^{\prime \prime}, a_{-i}\right)=0, T_{j} \in \boldsymbol{T}, T_{j} \notin \boldsymbol{A}_{i}} \omega_{T_{j}}\right)+ \\
U_{v_{i}}\left(a_{i}^{\prime}, a_{-i}\right)-U_{v_{i}}\left(a_{i}^{\prime \prime}, a_{-i}\right)
\end{gathered}
$$


Since the selections of other missiles are supposed to be constant during the local selection process in the potential game, thus in the formula above, we have

$$
\begin{gathered}
\sum_{T_{j} \in \boldsymbol{T}, T_{j} \notin \boldsymbol{A}_{i}} U_{T_{j}}\left(a_{i}^{\prime}, a_{-i}\right)- \\
\left.\lambda \cdot \sum_{n_{T_{j}}\left(a_{i}^{\prime}, a_{-i}\right)=0, T_{j} \in \boldsymbol{T}, T_{j} \notin \boldsymbol{A}_{i}} \omega_{T_{j}}\right)- \\
\left(\sum_{T_{j} \in \boldsymbol{T}, T_{j} \notin \boldsymbol{A}_{i}} U_{T_{j}}\left(a_{i}^{\prime \prime}, a_{-i}\right)-\right. \\
\left.\lambda \cdot \sum_{n_{T_{j}}\left(a_{i}^{\prime \prime}, a_{-i}\right)=0, T_{j} \in \boldsymbol{T}, T_{j} \notin \boldsymbol{A}_{i}} \omega_{T_{j}}\right)=0 .
\end{gathered}
$$

Then we obtain

$$
\begin{gathered}
U_{v_{i}}\left(a_{i}^{\prime}, a_{-i}\right)-U_{v_{i}}\left(a_{i}^{\prime \prime}, a_{-i}\right)>0 \Leftrightarrow \\
U_{g}\left(a_{i}^{\prime}, a_{-i}\right)-U_{g}\left(a_{i}^{\prime \prime}, a_{-i}\right)>0 .
\end{gathered}
$$

The constraint of the first mentioned individual utility function is difficult to meet in the process of the mission executed by MAF, so the proof of the convergence characteristic of the coordination mechanism is mainly for the second individual utility function which is appropriate for engineering application.

\section{Coordination mechanism}

In addition to the designed individual utility function and global utility function, the distributed decision based on the potential game also needs an appropriate coordination mechanism corresponding to the utility function to make the system converge to equilibrium. The quality of the coordination mechanism will directly affect the quality of the balance point. On the basis of spatial adaptive play (SAP), this paper introduces the idea of autonomous formation principles and constructs the coordination mechanism applicable to the dynamic target allocation of MAF. The main idea is the "cyclical selected SAP" (CSSAP) and "negotiation based on time division multiple address (TDMA) [30,31] protocol formation support network".

The basic rules of the coordination mechanism are described as follows.

In the SAP coordination mechanism [10], one missile is selected randomly to optimize the local target selection in each step, and inform the others the updated target through the support network. Assuming $\boldsymbol{a}(k-1)$ denotes the selection plan of all the missiles in the last step, the selection plan for the selected missile $\nu_{i}$ at the step $k$ is designed according to a specific probability distribution:

$$
\left\{\begin{array}{l}
\boldsymbol{p}_{i}(k) \in \boldsymbol{\Delta}\left(\left|\boldsymbol{A}_{i}\right|\right) \\
\Delta(n)=\left\{\boldsymbol{s} \in \mathbf{R}^{n} \mid \boldsymbol{s} \geqslant 0, \mathbf{1}^{\mathrm{T}} \boldsymbol{s}=1\right\}
\end{array}\right.
$$

where the probability distribution $p_{i}(k)$ can maximize

$$
\boldsymbol{p}_{i}^{\mathrm{T}}(k)\left[\begin{array}{c}
U_{v_{i}}\left(A_{i}^{1}, a_{-1}(k-1)\right) \\
\vdots \\
U_{v_{i}}\left(A_{i}^{\left|A_{i}\right|}, a_{-1}(k-1)\right)
\end{array}\right]+\tau H\left[\boldsymbol{p}_{i}(k)\right]
$$

where $H[\cdot]$ is the entropy function, and $\tau>0$ is a parameter used to adjust the randomness of the decision process. Thus, the above formula will reach maximum, only when $\boldsymbol{p}_{i}(k)$ is

$$
\boldsymbol{p}_{i}(k)=\sigma\left(\frac{1}{\tau}\left[\begin{array}{c}
U_{v_{i}}\left(A_{i}^{1}, a_{-1}(k-1)\right) \\
\vdots \\
U_{v_{i}}\left(A_{i}^{\left|A_{i}\right|}, a_{-1}(k-1)\right)
\end{array}\right]\right)
$$

where $\sigma(\cdot)$ is the logit or soft-max function as follows:

$$
\begin{gathered}
\sigma: \mathbf{R}^{n} \rightarrow \boldsymbol{\Delta}(n)=\text { logit or } \\
\text { soft }- \text { max function } \\
(\sigma(x))_{i}=\mathrm{e}^{x_{i}} /\left(\mathrm{e}^{x_{1}}+\cdots+\mathrm{e}^{x_{n}}\right) .
\end{gathered}
$$

Unlike the SAP in [10], the communication used in the formation support network in this paper is based on the AdHoc network with TDMA protocol. In each communication cycle, it is difficult to decide which missile's selection to be updated for a formation with distributed mechanism, so CSSAP is adopted. In this way, each missile is allowed to periodically update its selection, and although a certain degree of randomness is given up, the system could meet the requirements of SAP coordination mechanism as well. That means each missile's selection will be updated once in $n$ cycles, according to the missile number $i(i \in\{1,2, \ldots, n\})$.

Moreover, in order to speed up the convergence rate of CSSAP, the global correction of the uncertain information is added based on the formation support network service quality cognition when each missile updates the local target. Assume that the formation support network has relative complete service quality and cognitive ability, each node can learn its own network service quality (mainly consider packet loss rate in this paper). Then when a missile needs to update its own target in order, there is a probability $p\left(\kappa_{i}\right)$ to abandon the target update because of the packet loss rate. To sum up, the improved SAP coordination mechanism used in this paper is as follows.

The target of the missile $v_{i}$ is updated during the number $n \cdot i$ communication cycle, according to the following mechanism.

If $p>\kappa_{i}$, then select $a_{i}(k)$ by the probability distribution:

$$
\boldsymbol{p}_{i}(k)=\sigma\left(\frac{1}{\tau}\left[\begin{array}{c}
U_{v_{i}}\left(A_{i}^{1}, a_{-i}(k-1)\right) \\
\vdots \\
U_{v_{i}}\left(A_{i}^{\left|A_{i}\right|}, a_{-i}(k-1)\right)
\end{array}\right]\right)
$$


where $\boldsymbol{p}_{i}(k) \in \boldsymbol{\Delta}\left(\left|\boldsymbol{A}_{i}\right|\right)$, and if $p \leqslant \kappa_{i}$, then we get $a_{i}(k)=a_{i}(k-1)$ directly.

The following proves that this coordination mechanism can make the potential game converge to a balance point. Firstly, Theorem 6.1 in the literature [32] is introduced to prove that $\lim _{\tau \downarrow 0} \lim _{\boldsymbol{\kappa} \downarrow 0} \lim _{k \rightarrow \infty} \operatorname{Prob}\{\boldsymbol{a}(k)$ is a potential game allocation $\}=1$.

Lemma 1 Let $G$ be a symmetric potential game with potential energy function $\rho$. And set $\Gamma$ as a finite weighted graph. For each $\beta>0$, the spatial adaptability process $P^{\Gamma, \beta}$ has a unique stable distribution as

$$
\mu^{\Gamma, \beta}(x)=\mathrm{e}^{\beta \rho^{*}(x)} / \sum_{z \in \Xi} \mathrm{e}^{\beta \rho^{*}(z)}
$$

and the random stable state of the spatial game is the state that maximizes $\rho^{*}(x)$.

According to Lemma 1, the improved SAP coordination mechanism in this paper has introduced an irreducible Markov process. The state space is $A$, and the state of step $k$ is expressed as $\boldsymbol{a}(k)$. The transfer frequency of this Markov process state will converge to a unique stable distribution, which can be written as follows, as given in Lemma 1:

$$
\mu^{\tau}(\boldsymbol{a})=\frac{\mathrm{e}^{(1 / \tau) U_{g}(\boldsymbol{a})}}{\sum_{\overline{\boldsymbol{a}} \in \boldsymbol{A}} \mathrm{e}^{(1 / \tau) U_{g}(\overline{\boldsymbol{a}})}}, \quad \forall \boldsymbol{a} \in \boldsymbol{A}
$$

and the detailed balance condition is

$$
\begin{gathered}
\mu^{\tau}(\boldsymbol{a}) \operatorname{Prob}\{\boldsymbol{a} \rightarrow \boldsymbol{b}\}= \\
\mu^{\tau}(\boldsymbol{b}) \operatorname{Prob}\{\boldsymbol{b} \rightarrow \boldsymbol{a}\}, \quad \forall \boldsymbol{a}, \boldsymbol{b} \in \boldsymbol{A} .
\end{gathered}
$$

As in this coordination mechanism, the nodes are periodically selected to update, so it only needs to prove the balance condition of the two states $\boldsymbol{a}, \boldsymbol{b} \in \boldsymbol{A}$, inside which there is only one different element, for example the specific $\boldsymbol{a}, \boldsymbol{b} \in \boldsymbol{A}, a_{i} \neq b_{i}, a_{-i}=b_{-i}$, and the balance condition equation needed to be proved could be obtained as follows:

$$
\begin{gathered}
\operatorname{Prob}\{\boldsymbol{a} \rightarrow \boldsymbol{b}\}= \\
n_{v} \sum_{\left(a^{1}, \ldots, a^{n_{i}}\right) \in S(\boldsymbol{a}, \boldsymbol{b})} \frac{1}{\left(\left|\boldsymbol{A}_{i}\right|-1\right) \cdots\left(\left|\boldsymbol{A}_{i}\right|-n_{i}\right)} . \\
\frac{\mathrm{e}^{(1 / \tau) U_{v_{i}}(\boldsymbol{b})}}{\sum_{j=0}^{n_{i}} \mathrm{e}^{(1 / \tau) U_{v_{i}}\left(a^{j}\right)}} \\
\begin{array}{c}
S(\boldsymbol{a}, \boldsymbol{b})=\left\{\left(a^{1}, \ldots, a^{n_{i}}\right) \in A^{n_{i}+1}:\right. \\
\left(a_{-i}^{j}=a_{-i}, \forall j\right),\left(a^{0}=a\right),\left(a^{j}=b\right) \\
\left.\left(\boldsymbol{a}^{j} \neq \boldsymbol{b}^{m}, \forall j, m\right)\right\} .
\end{array}
\end{gathered}
$$

Then (19) is derived:

$$
\begin{gathered}
\frac{\operatorname{Prob}\{\boldsymbol{a} \rightarrow \boldsymbol{b}\}}{\operatorname{Prob}\{\boldsymbol{b} \rightarrow \boldsymbol{a}\}}=\mathrm{e}^{(1 / \tau)\left[U_{v_{i}}(\boldsymbol{b})-U_{v_{i}}(\boldsymbol{a})\right]}= \\
\mathrm{e}^{(1 / \tau)\left[U_{g}(\boldsymbol{b})-U_{g}(\boldsymbol{a})\right]}=\frac{\mu^{\tau}(\boldsymbol{b})}{\mu^{\tau}(\boldsymbol{a})} .
\end{gathered}
$$

It can be seen that the coordination mechanism can have a higher probability to make the autonomous formation converge to balance in a certain network packet loss, and this target dynamic allocation method using the potential game has the characteristics of low computing and communication burden, and is suitable for engineering applications.

\section{Dominating value for the missile to the target}

In this paper, the distributed allocation problem during an anti-ship missiles autonomous formation executing cooperative combat task is taken as an example, and the non-parametric method is used to calculate the dominating value for the missile to the target. The dominant function is constructed according to the angle, distance, velocity and missile capture probability, radiation source and other parameters [33].

(i) Missile-target angle dominance

Assume that the relative motion relationship between the missile and the target is shown in Fig. 2, where $M$ is the missile, $V_{M}$ denotes the missile's velocity, $T$ is the target, and $V_{T}$ denotes the target's velocity, so it can be seen, $r$ is the distance between the missile and the target, and LOS is the line of sight between the missile and the target, $\eta$ is the angle between $V_{M}$ and $L O S, \eta_{T}$ is the angle between $V_{T}$ and $L O S$.

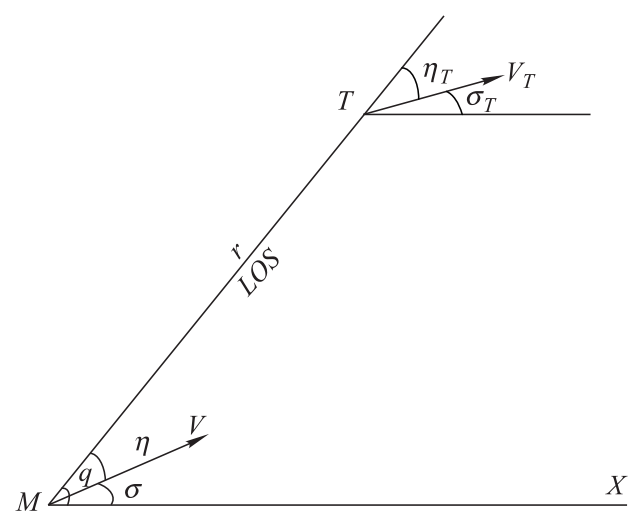

Fig. 2 Relative motion relationship between the missile and the target

When the velocity of the missile is closer to the $L O S$, the missile is easier to attack the target. In other words, 
when $\eta$ equals zero, the missile has the biggest angle advantages on the target as

$$
S_{\varphi}=\mathrm{e}^{-\left|\frac{\eta}{a \pi r}\right|}
$$

where $a$ is a variable parameter varing with the distance between the missile and the target. Here we assume parameter $a$ has the proportional relationship with $r$, because the bigger $r$ the smaller the impact on $S_{\varphi}$ from angle $\eta$.

(ii) Missile-target distance dominance

Assume that the seeking range of the missile seeker is $R_{\min }-R_{\max } \cdot R_{\min }$ and $R_{\max }$ are respectively the seeker near and far boundaries. The distance dominance is usually judged to be very small when $r \rightarrow R_{\text {max }}$ or $r \rightarrow R_{\min }$, while the distance dominance will be the greatest when $r=\left(R_{\min }+R_{\max }\right) / 2$. Therefore, the distance advantage function can be constructed as

$$
S_{r}=\left(\mathrm{e}^{-\left|\frac{r}{R_{0}}-1\right|}+\mathrm{e}^{-|\Delta z|}\right) / 2
$$

where $R_{0}=\left(R_{\min }+R_{\max }\right) / 2$.

(iii) Missile-target velocity dominance

The ratio of the missile's velocity to the target's velocity is added to the dominant function. In most cases, the missile can hit the target, only when the velocity of the missile is bigger than the target's velocity. Therefore, the following formula could be used to construct the velocity dominant function between the missile and the target:

$$
S_{v}=1.0-V_{T} / V_{M} \text {. }
$$

(iv) Missile-target matching dominance

Different seekers have different detection capabilities for different targets. Thus the seekers' detection ability information will be normalized, that is to compare every seeker's probing distance to the target with the maximum probing distance of the same kind seeker. In this way, the difference between the different seekers in the obtained matching information is covered up, and the matching dominant function value of the same seeker is always less than or equal to 1 , which is convenient for subsequent calculation. The missile-target matching dominance is

$$
S_{s}=R_{m t} / R_{z}
$$

where $R_{m t}$ is the detectable distance for the seeker to the current target, and $R_{z}$ is the maximum effective probing distance in the same kind of seeker.

(v) Synthetical missile-target dominance

According to the dominant functions constructed above, the synthetical dominant function between a missile and its target could be constructed as

$$
S=S_{\varphi}\left(C_{1} \times S_{R}+C_{2} \times S_{v}\right)+C_{3} \times S_{s}
$$

where $C_{1}, C_{2}, C_{3}$ are weight coefficients, $0 \leqslant C_{i} \leqslant 1$, and $\sum_{i=1}^{3} C_{i}=1$, whose values are decided by the effect of each dominance to the synthetical missile-target dominance.

\section{Simulation}

In order to facilitate the study of MAF cooperative guidance and control technology, simultaneously to simulate and verify the technology related to the sub-system, an integrated digital simulation platform is built by the research team members together. The system is built on Vega Prime and Microsoft Visual Studio 2005 to establish a nonlinear six degree-of-freedom model of a certain type of anti-ship missile simulation data. The MAF executes the task in the battlefield shown in Fig. 3 and the simulation platform has the following functions: battlefield situation real-time display and record, complete sub-system function and high precision simulation.

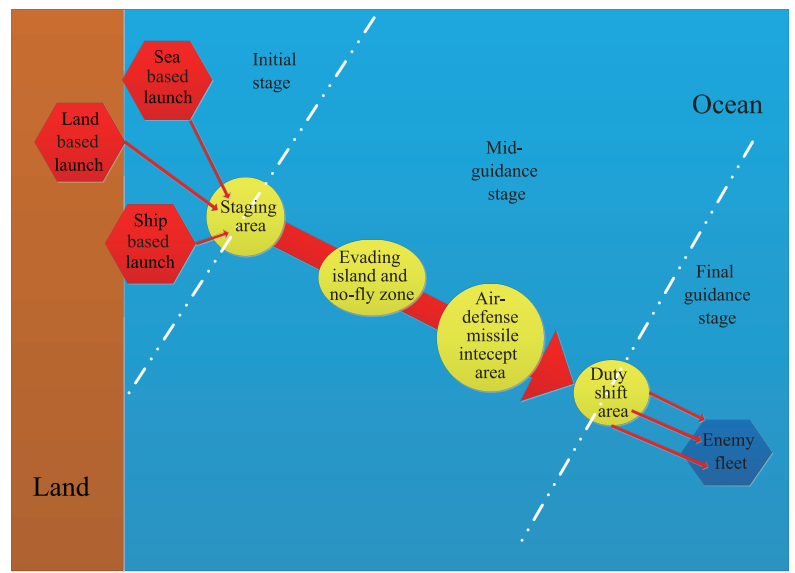

Fig. 3 Typical warfare scenario diagram

The program framework flow chart of the simulation platform is shown in Fig. 4. In real-time mode, the simulation system initialization in accordance with the battle hypothesis and each function module initialization are performed firstly, and then the multi-thread is created and initialized in order to make each combat unit be assigned in a separate thread, so that the simulation could process more rationally. In this way, the real-time simulation of attack and defense is performed, as each thread runs in parallel. The visual cycle display is in the control of the simulation while battle assessment and data manipulation are conducted at last.

In the integrated digital simulation platform, the distributed decision method proposed in this paper is applied to the MAF dynamic target allocation during the handover guidance stage between midcourse and final guidance. The allocation result of distributed decision and the change 
of the utility function value of the potential game can be monitored in real-time on the platform.

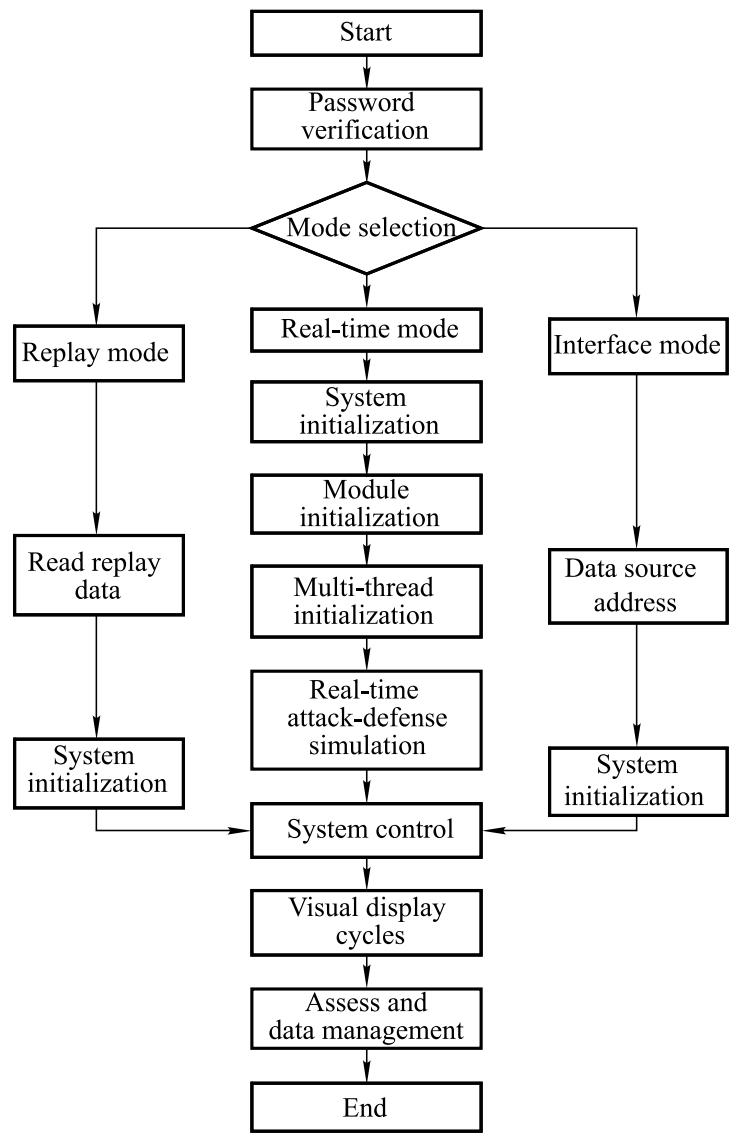

Fig. 4 Typical warfare scenario diagram

And the change of the utility function value can also be obtained when the formation works with different net- work service qualities. The simulation focuses on the study of the distributed decision method based on the potential game and formation principles. Therefore, assumptions and instructions for the MAF's cooperative guidance and control capability and the functions of the system are given as follows:

(i) The anti-ship missile's velocity is $0.45-3.0 \mathrm{Ma}$, the overload capacity is less than $10 \mathrm{~g}$, the cruising altitude is $15000 \mathrm{~m}$, and the flight height beyond the sea can be as low as $10 \mathrm{~m}$; (ii) After the seeker is turned on and the missile flies into the handover guidance stage, it is considered that the target information has been fused and the target characteristic is unchanged; (iii) The optional target set of the missiles, the position and the velocity dominance of the missiles to the targets, the target threat and other parameters keep in constant during the handover guidance stage; (iv) Each missile involved in the potential game is constantly sending its target selected using the coordination mechanism at the moment, and receiving the choices of other missiles which cannot be guaranteed a real-time receiving with network packet loss; (v) Assume that each missile can accurately obtain the dominating value for the missiles assigned to every target in its target set, where we consider that the information is relative accurate because the distance is not far, and there is uncertainty to use the other missiles' dominating values to their targets; (vi) Assume that each missile has been informed of the network service quality (mainly the set packet loss rate) of all the formation members through the support network, but it is random whether each packet data is lost. The simulation schematic diagram is as Fig. 5.

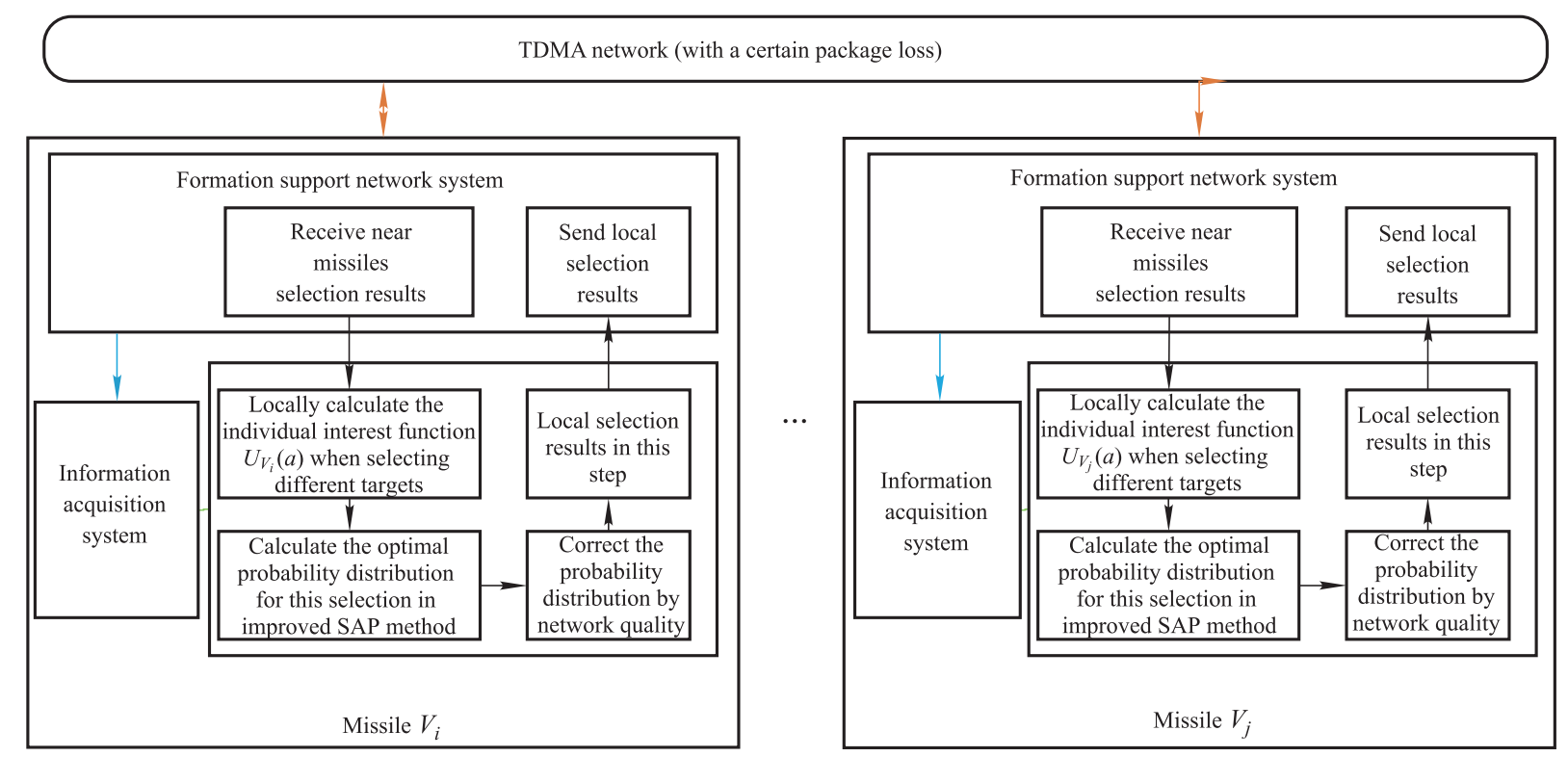

Fig. 5 Simulation schematic diagram 
Fig. 6 -Fig. 9 show the experimental scenes in the integrated digital simulation platform, vividly showing the whole scenes of the battle.

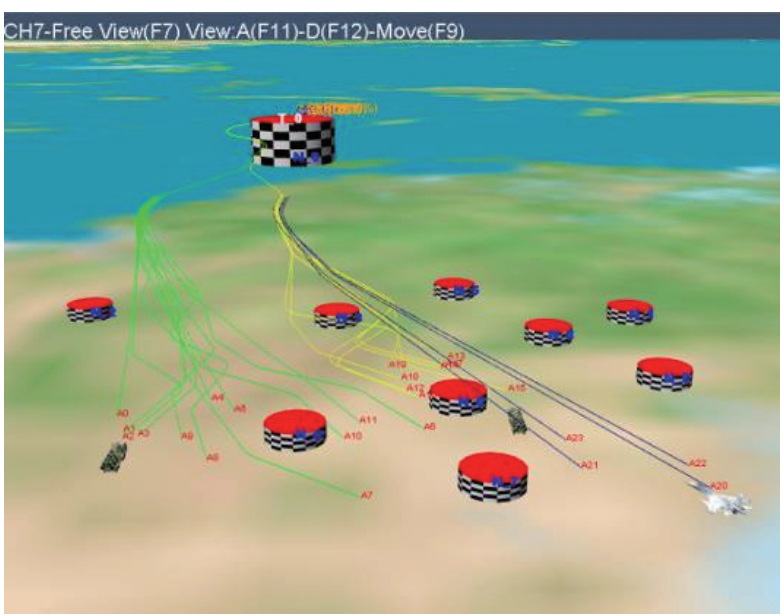

Fig. 6 Simulation initial state scene

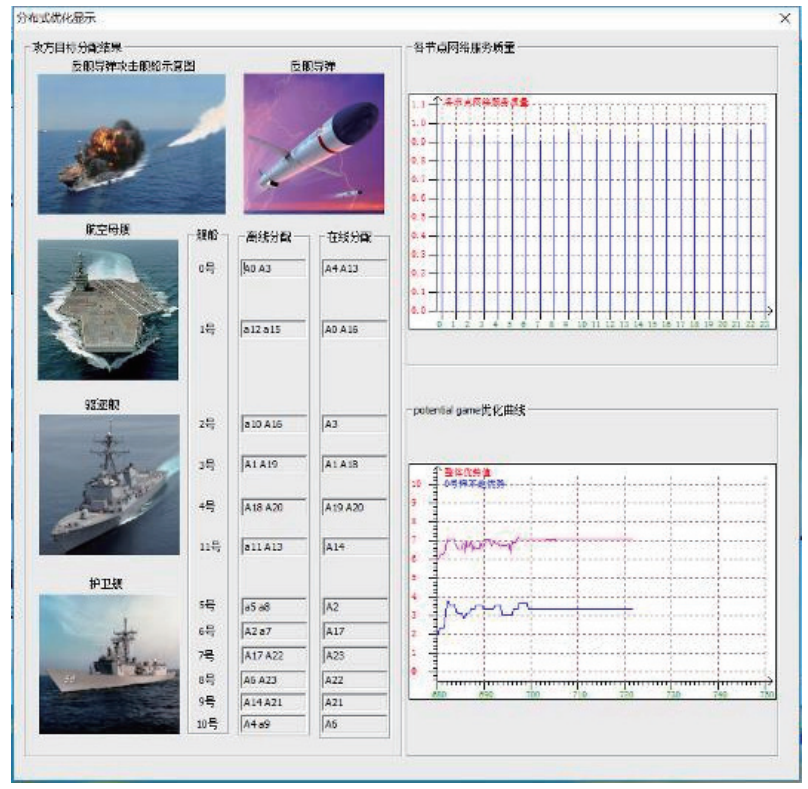

Fig. 7 Distributed decision process

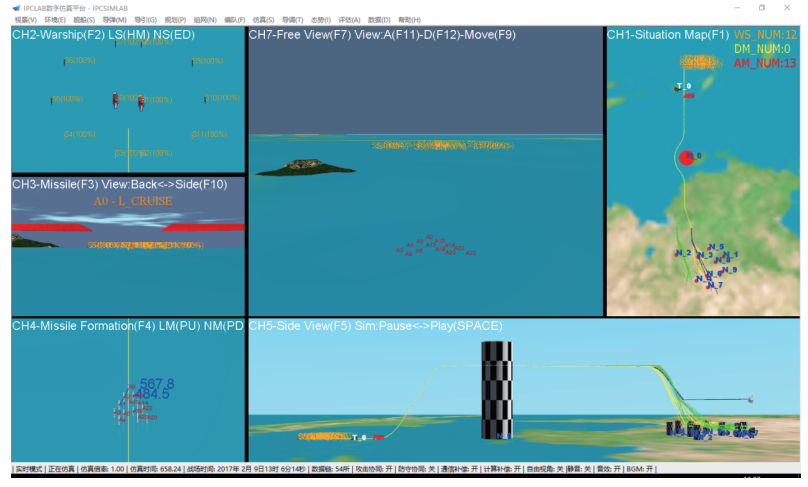

Fig. 8 Low-altitude cruise state before handover guidance

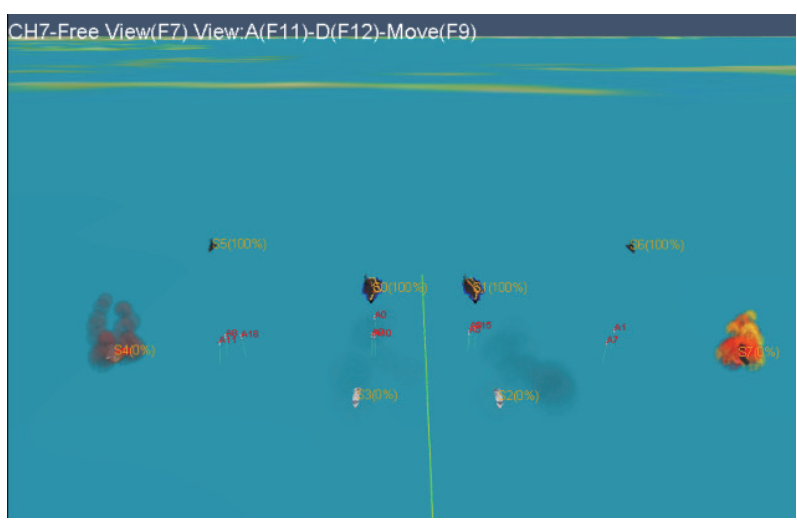

Fig. 9 Visual scene of targets destruction

The distributed decision display interface of the simulation platform can monitor the allocation results of the MAF and the numerical change curves of the global and individual utility function values. From Fig. 10, we can see the convergence trend of the potential game and the optimization of the dynamic target allocation in the progress of the handover guidance.

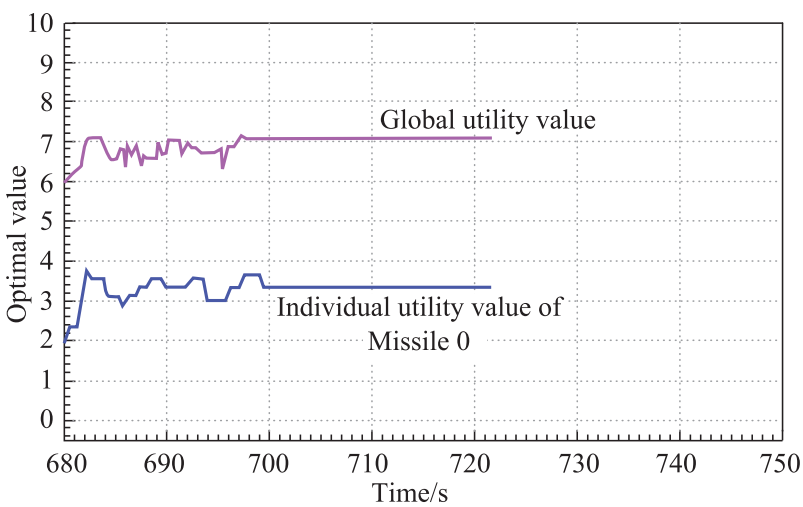

Fig. 10 Change curve of utility function

It can be seen from Fig. 10 that the curve trend of the individual utility function and the global utility function is basically the same, and the utility function value of the final allocation result is obviously higher than the initial value. In the actual optimization process, the randomness parameter $\tau$ and the packet loss rate $\kappa$ of the support network are not the infinitesimal that tends to zero, so the potential game result will have random fluctuation. However, in engineering application a significant optimization effect for the distributed decision could be ensured within a specific decision time.

\section{Conclusions}

In this paper, a distributed decision method for MAF based on the potential game and formation principles is proposed. The individual utility function, global utility function and 
improved SAP coordination mechanism based on the potential game and formation principles are constructed. The designed coordination mechanism takes into account the practical application of the autonomous formation, and introduces the idea of "CSSAP" and the idea of "macroregulation based on the formation support network cognition". At last the effectiveness of the method is verified on the MAF cooperative guidance and control technology integrated digital simulation platform. It can be seen that this method can guarantee a large-scale high-dynamic missiles autonomous formation to achieve the distributed dynamic target allocation with a certain information uncertainty and a packet loss in network communication.

\section{References}

[1] SHIMA T, RASMUSSEN S. UAV cooperative decision and control: challenges and practical approaches. IEEE Control Systems Magazine, 2010, 30(2): 104- 107.

[2] OLFATI-SABER R. Flocking for multi-agent dynamic systems: algorithms and theory. IEEE Trans. on Automatic Control, 2006, 51(3): $401-420$.

[3] AHUJA R K, KUMAR A, JHA K, et al. Exact and heuristic methods for the weapon-target assignment problem. Operations Research, 2007, 55(6): 1136-1146.

[4] GINTIS H. Game theory evolving: a problem-centered introduction to modeling srtrategic interaction. Princeton: Princeton University Press, 2009.

[5] FUDENBERG D, TIROLE J. Game theory. Cambridge: MIT Press, 1991.

[6] LUCE R, RAIFFA H. Games and decisions: introduction and critical survey. New York: Dover, 1989.

[7] MONDERER D, SHAPLEY L S. Potential games. Games and Economic Behavior, 1996, 14: 124-143.

[8] MARDEN J R, ARSLAN G, SHAMMA J S. Cooperative control and potential games. IEEE Trans. on Systems, Man, and Cybernetics, Part B, 2009, 39: 1393 - 1407.

[9] ZHANG J, QI D, ZHAO G. A new game model for distributed optimization problems with directed communication topologies. Neurocomputing, 2015, 148: 278-287.

[10] ARSLAN G, MARDEN J R, SHAMMA J S. Autonomous air vehicle target assignment: a game theoretical formulation. Journal of Dynamic Systems, Measurement, and Control, 2007, 129: $584-596$.

[11] LI P, DUAN H. A potential game approach to multiple UAV cooperative search and surveillance. Aerospace Science \& Technology, 2017, 68: 403-415.

[12] MARDEN J R. State based potential games. Automatica, 2012, 48: 3075-3088.

[13] JOHARI R, MANNOR S, SITSIKLIS J T. Efficiency loss in a network resource allocation game: the case of elastic supply. IEEE Trans. on Automatic Control, 2005, 50 (10): $1712-$ 1724.

[14] YOUNG H P. Learning by trial and error. Games and Economic Behavior, 2009, 65: 626-643.

[15] LI N, MARDEN J R. Designing games for distributed optimization. IEEE Journal of Selected Topics in Signal Processing, 2011, 7(2): 2434-2440.
[16] WU S. Cooperative guidance \& control of missiles autonomous formation. Beijing: National Defense Industry Press, 2015. (in Chinese)

[17] WEN Y, WU S, LIU W, et al. A collision forecast and coordination algorithm in configuration control of missile autonomous formation. IEEE Access, 2017, 5: 1188-1199.

[18] WU S. Cooperative flight control system. Beijing: Science Press, 2015. (in Chinese)

[19] JIA X, WU S T, WEN Y M, et al. Assessment method for comprehensive combat effectiveness of missile autonomous formation. Journal of Beijing University of Aeronautics \& Astronautics, 2017, 43(5): 1013 - 1022. (in Chinese)

[20] LIU W, WU S, WU X. Relative navigation of missile formation and INS error correction methods. Proc. of the Control \& Decision Conference, 2017, DOI: 10.1109/CCDC.2017.7978763.

[21] KE M, XU Y, ANPALAGAN A, et al. Distributed TOA-based positioning in wireless sensor networks: a potential game approach. IEEE Communications Letters, 2017, 22(2): 316319.

[22] LEE S J, PARK S S, CHOI H L. Potential game-based nonmyopic sensor network planning for multi-target tracking. IEEE Access, 2018, 6: 79245 - 79257.

[23] KE M X, LI D, TIAN S W, et al. Distributed power allocation for wireless sensor network localization: a potential game approach. Sensors, 2018, 18(5): 1480.

[24] RAN X M, FANG D L. Distributed sensor allocation algorithm for target tracking based on potential game. Journal of Electronics and Information Technology, 2017, 39(10): 2748 2754. (in Chinese)

[25] RAMASWAMY V, REDDY V, SHAKKOTTAI S, et al. Multipath wireless network coding: an augmented potential game perspective. IEEE/ACM Trans. on Networking, 2014, 22(1): $217-229$.

[26] VAN NGUYEN C, HOANG P H, KIM H, et al. Distributed learning in a multi-agent potential game. Proc. of the 17th International Conference on Control, 2017: 266-271.

[27] TATARENKO T. Stochastic payoff-based learning in multiagent systems modeled by means of potential games. Proc. of the IEEE 55th Conference on Decision and Control, 2016, DOI: 10.1109/CDC.2016.7799081.

[28] LIN Z, LIU H T. Consensus based on learning game theory with a UAV rendezvous application. Chinese Journal of Aeronautics, 2015, 28(1): $191-199$.

[29] GOTO T, HATANAKA T, FUJITA M. Payoff-based inhomogeneous partially irrational play for potential game theoretic cooperative control of multi-agent systems. eprint arXiv, 2011: 1107.4838 .

[30] ZHANG R, CHENG X, YANG L, et al. A novel centralized TDMA-based scheduling protocol for vehicular networks. IEEE Trans. on Intelligent Transportation Systems, 2015, 16(1): $411-416$.

[31] LAM S S. Delay analysis of a time division multiple access (TDMA) channel. IEEE Trans. on Communications, 1978, 25(11): $1489-1494$.

[32] YOUNG H P. Strategic learning and its limits. Oxford: Oxford University Press, 2005.

[33] ZHANG B C, PENG C, YU X R. Cooperative target assignment algorithm of multiple missile based on ant colony opti- 
mization. Journal of Projectiles, Rockets, Missiles and Guidance, 2012, 32(4): 69-73. (in Chinese)

\section{Biographies}

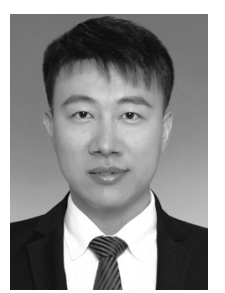

JIA Xiang was born in 1989. He received his Ph.D. degree from Beihang University, and now is working for Beijing Institute of Electronic Engineering. He mainly studies the decision and management system of missiles autonomous formation.

E-mail: 1hfy607@163.com

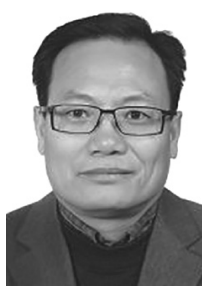

WU Sentang is a professor of automation science and electrical engineering, and a doctoral tutor in Beihang University. In February 1992, he obtained his engineering doctorate of dynamics, ballistics and aircraft motion control system in Soviet Kiev Aeronautical Engineering Institute (now Ukraine National Aviation University). He is a member of the navy missile expert group for National Defense Ba- sic Research Institute. He mainly studies the theory and application of nonlinear stochastic systems, computer information processing and control, aircraft cooperative control and precision guidance and so on.

E-mail: woost@sina.com

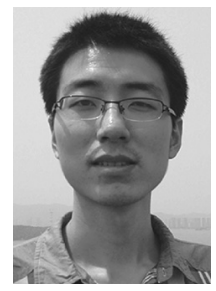

WEN Yongming was born in 1988. He received his Ph.D. degree from Beihang University. He mainly studies the aircraft autonomous formation flight control system.

E-mail: wenyongming_buaa@foxmail.com

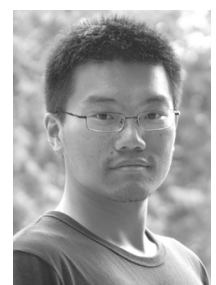

YAO Zheng was born in 1990. He is a Ph.D. student in Beihang University. He mainly studies the decision and management system of heterogeneous multi-agent system and morphing aircraft.

E-mail: i_loveinter@126.com 